\title{
Effects of Drought Stress on Growth of Lettuce Seedlings
}

\author{
Yu Zhang, Qi He, Fangjie Xie and Mengyao Li* \\ College of Horticulture, Sichuan Agricultural University, Chengdu, China \\ *Corresponding author e-mail: limy@sicau.edu.cn
}

Keywords: Lettuce, drought, chlorophyll.

\begin{abstract}
The physiology and biochemical indices include the root length, the root cap ratio, the water content of blade, chlorophyll content, soluble protein content, and soluble sugar content were measured to analyzed the effects of different degrees of drought stress on lettuce seedlings. The data showed that the root length of lettuce increased with the increase of drought stress treatment time, and the drought degree is more serious, the growth of root length and the root cap ratio are more significant. The water content and chlorophyll content of lettuce decreased more obviously the drought duration; the soluble sugar content and soluble protein content of lettuce increased firstly and then decreased, and the more serious the drought was, the greater the effect was.
\end{abstract}

\section{Introduction}

Lettuce (Lactuca sativa. L), an annual or biennial species belongs to the Composite family, is a low-sugar and low-fat vegetable widely used in the world [1]. There is a great demand for water in lettuce cultivation, and the water has become an important factor restricting lettuce production [2]. Studying the response mechanism of the morphological and physiological of lettuce under drought stress, and clarifying the important physiological indicators related to drought resistance, which can provide a reference for basic research on the selection and cultivation of drought resistant lettuce varieties [3].

Water is considered as a one of determinant factor that affects plant growth. The physiological and morphological of plants will change in response to drought stress. Leaves would wither under water shortage, and severe water shortage would lead to plant death [4-5]. Studies have shown that some substances like soluble sugar, soluble protein, betaine and proline have a very significant change in their content during drought stress. These substances act as permeability regulating substances which can help to maintain cell water potential and allow cells to complete the normal physiological metabolism [6-8].

In this experiment, different degrees of drought stress treatment were carried out to analyze the changes of external phenotypes and physiological indexes of lettuce seedlings. The relationship between the physiological parameters and the drought resistance of lettuce was further investigated, which can provide a reference for further research on the response mechanism to drought stress and screening drought-tolerant varieties of lettuce.

\section{Materials and methods}

\subsection{Plant materials, growth conditions, and experimental treatments}

Lettuce seedlings (Lactuca sativa. L, cultivar Italy lettuce) with four leaves and one heart were used as experiment materials. Plants were irrigated with different PEG-6000 solutions (0, 20\%, and 40\%) and samples were harvest at 1, 3, 5, and $7 \mathrm{~d}$ after treatment. Three independent biological replicates were used in all experiments.

\subsection{Data measurement and analysis}

The root length and plant height were measured with vernier caliper and the weight of plant was measured with electronic scales. Soluble protein content was determined by Coomassie brilliant blue 
method, and soluble sugar content was measured with anthrone colorimetric method. Chlorophyll content was measured using spectrophotometer software.

\section{Results}

\subsection{Effects of drought stress on leaf water content, root system and root shoot ratio of lettuce}

As shown in Figure 1, with the increase of treatment days, the content of leaf water from three groups showed a state of gradual decrease. After 7 days, the $20 \%$ treatment decreased with $12.7 \%$ compared with CK, while the $40 \%$ treatment decreased with $18.6 \%$ compared with CK. The results showed that the water content of lettuce leaves was decreasing under drought stress, and the reduction is intensified with the continuous treatment time and processing concentration.

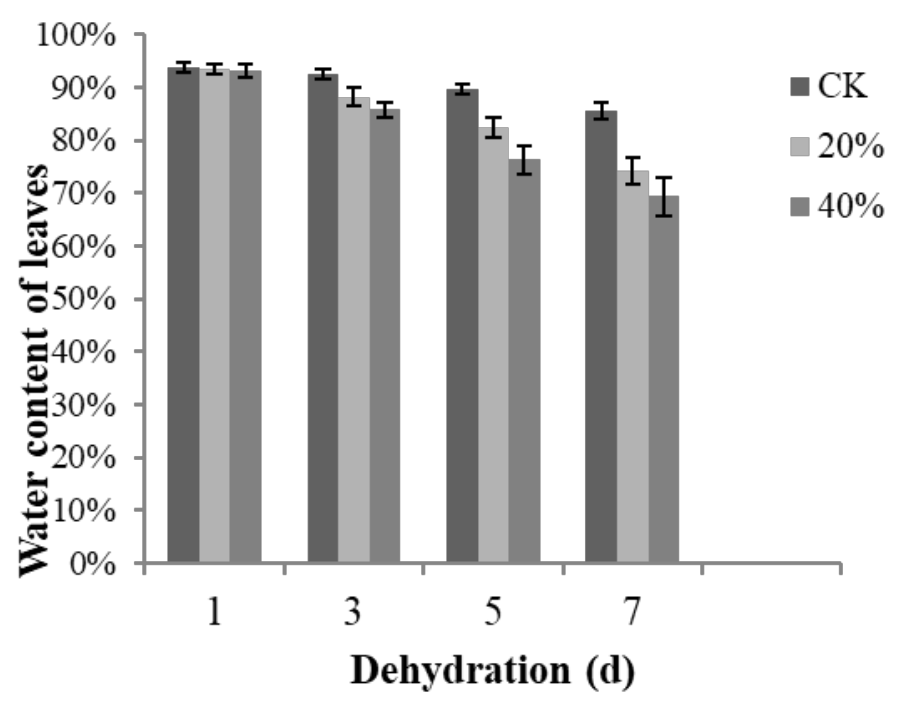

Figure 1. Effect of drought on water content of lettuce seedling leaves.

Plants will expand their water absorption area through the extension of root system under drought stress. As shown in Figure 2(a), with the extension of treatment time, the root length of the groups of treatment showed a trend of increasing, and the root length of the high concentration PEG6000 treatment increased more significantly. After 7 days, the root length of $20 \%$ treated plants increased $32.3 \%$ compared with CK, and that of $40 \%$ treated plants increased $41.2 \%$ compared with CK. The differences of the final root length of lettuce among the three groups were significant. As can be seen from Figure 2(b), the root-cap ratio under fresh weight of the three groups also showed an increasing trend. After 7 days, the root-shoot ratio of $40 \%$ treatment increased $26.3 \%$ compared to CK, and $20 \%$ treatment increased $14.4 \%$. This shows that under drought stress, the growth of lettuce roots is promoted, while the growth of above-ground parts is inhibited, and with the increase of stress time, the degree of inhibition is more significant.

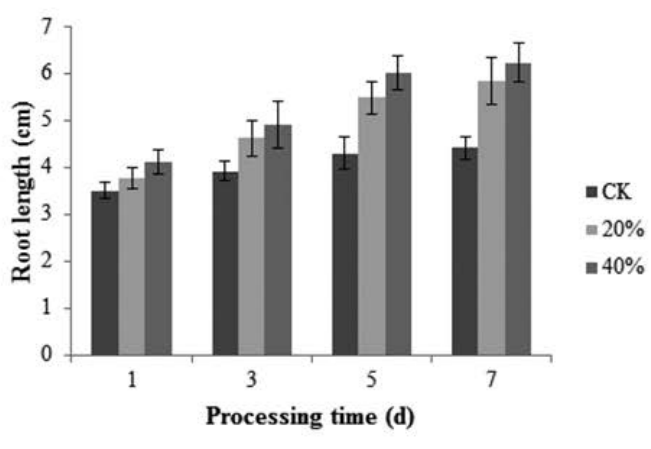

a

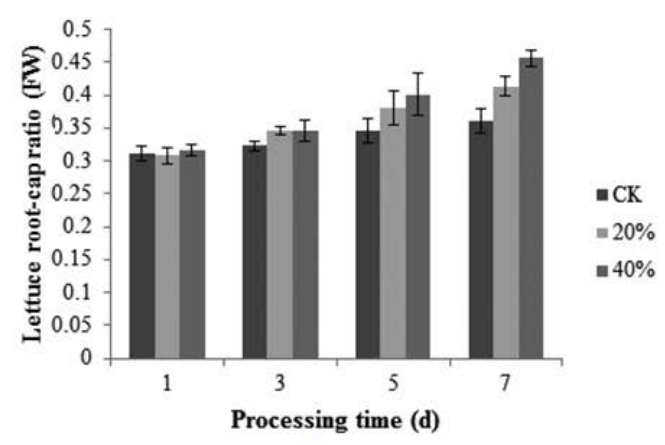

b

Figure 2. Effects of drought stress on root length and root-cap ratio of lettuce seedlings. 


\subsection{Effect of drought on soluble protein and soluble sugar in lettuce}

As illustrated in Figure 3(a), the soluble protein content of the two groups of stress treatments showed a state of continuous increase and then began to decrease five days before treatment, while CK continued to increase during the seven days of treatment. After one day of treatment, plants with 40\% PEG6000 treatment had a significant increase in the content of soluble protein, while plants with $20 \%$ treatment had no significant change. After five days, the two groups of stress treatment reached a peak, when compared with CK, 20\% treatment increased $20.8 \%$ and $40 \%$ treatment increased $30.7 \%$, respectively. The result showed that stress can promote the synthesis of soluble proteins in lettuce, and the greater the intensity of stress treatment, the greater the increase in the early stage.

The soluble sugar content of the two groups showed a trend of increasing before five days and then decreasing, while CK was increasing during seven days. There was a significant difference among three groups after three days, with $40 \%$ treatment $>20 \%$ treatment $>$ CK. After five days of treatment, the soluble sugar content in both groups had a peak. The $20 \%$ treatment increased $25.5 \%$ compared with CK, and the $40 \%$ treatment increased $25.6 \%$ compared with CK. After seven days of treatment, the stress treatment of the two groups showed a decreasing trend. This indicates that in the early stage of drought stress, stress can effectively promote the biosynthesis and transformation of soluble sugar, with the increase of treatment time, soluble sugar metabolism consumption increased, and the synthesis pathway was blocked, and its content began to decrease.

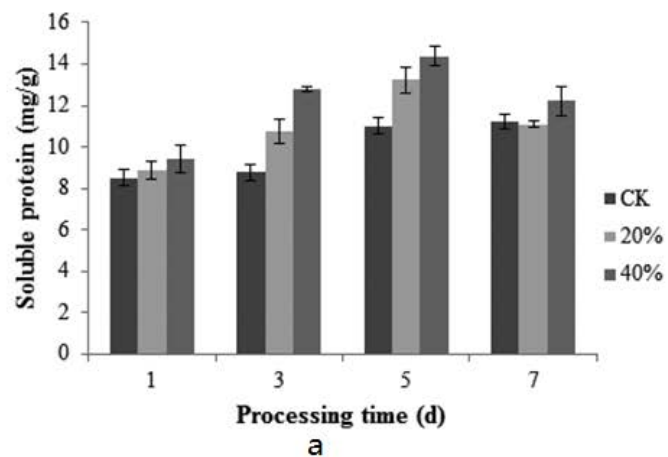

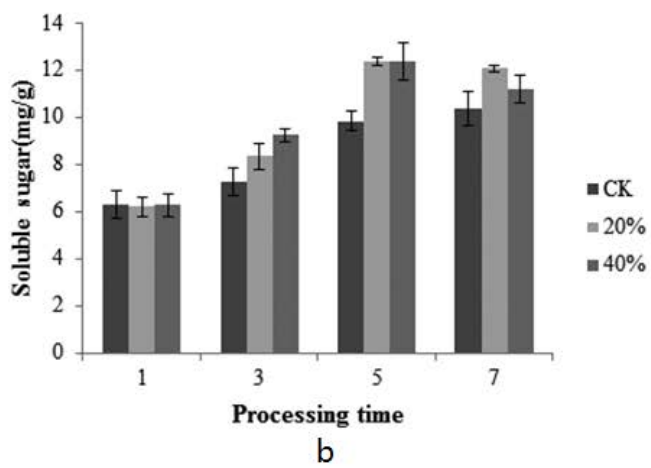

Figure 3. Effects of drought stress on soluble protein and soluble sugar in lettuce.

\subsection{Effects of drought on the content of chlorophyll in lettuce leaves}

As shown in Figure 4, the chlorophyll content of three groups of lettuce leaves showed a declining state in the seven days of treatment. After one day of treatment, the stress treatment in the two treatment groups decreased compared with CK, but the decrease was not significant. After five days, both of treatment groups began to decrease significantly. After seven days, the chlorophyll content in the leaves of the three groups was significantly different, the $40 \%$ treatment was 0.432 $\mathrm{mg} / \mathrm{g}, 17.2 \%$ lower than CK, and the $20 \%$ treatment was $0.477 \mathrm{mg} / \mathrm{g}, 8.61 \%$ lower than CK. The chlorophyll content of lettuce leaves decreased with the prolongation of the stress time, and the more severe the stress, the greater the reduction. 


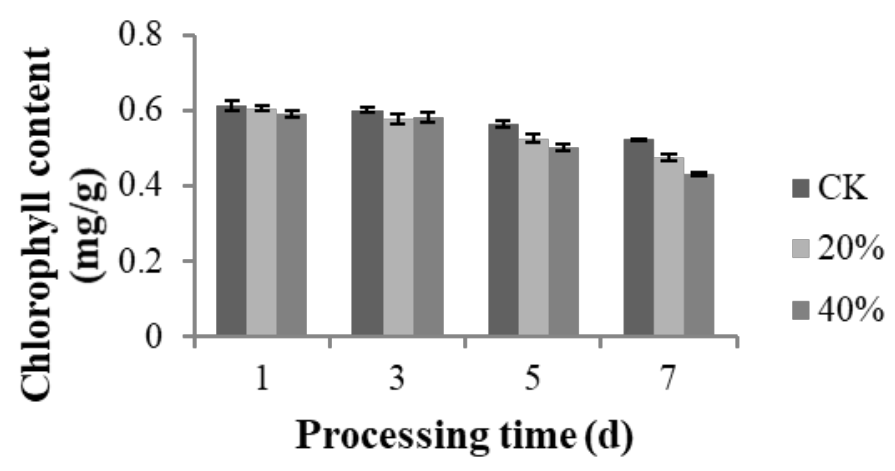

Figure 4. Effect of drought stress on chlorophyll content of lettuce seedling leaves.

\section{Discussion and conclusion}

The results showed that the root length and root-cap ratio of lettuce seedlings were increasing under drought stress, while leaf water content showed a decreasing trend. It shows that to a certain extent, drought stress can promote the elongation and growth of the root system and increase the water absorption area of the root system. However, it will inhibit the growth on the ground, causing the water content of the leaves above ground to continue to decline, affecting its growth and development, and the more severe the stress, the more significant the promotion of the underground part and the suppression of the above ground part.

Chlorophyll is an important substrate for plant photosynthesis and an important guarantee for normal growth and development of plants [9-10]. The loss rate of Chlorophyll under drought stress can be used as an important reference index for measuring plant injury rate. Moreover, the chlorophyll content is closely related to the water status of the plant. Water is an important substance for chlorophyll synthesis. The original chlorophyll will also be broken down in a water-deficient environment [11-13].The change curve of chlorophyll in this experiment is consistent with the change curve of leaf water content, which confirms the close relationship between plant water status and chlorophyll. Under drought stress, the chlorophyll content of lettuce seedlings continued to decrease, and the more severe the stress, the more significant the chlorophyll content decreased, indicating that the lettuce seedlings were not resistant to drought. Drought stress at the seedling stage will quickly affect the chlorophyll content, which in turn affects its photosynthesis and eventually causes its growth and development to be hindered.

As osmotic adjustment substances, the contents of soluble protein and soluble sugar increased first and then decreased in this study. The result indicating that in the early stage of drought stress, the soluble protein and soluble sugar could maintain the cell water potential and the intracellular water state to ensure normal physiological metabolism. However, with the extension of drought stress, the synthesis mechanisms of protein and sugar were blocked. The path of soluble sugar synthesis is blocked, and the content of the two gradually decreases [14].

The growth and development of lettuce seedlings are greatly affected under drought stress. The water content and chlorophyll content of leaves decreased significantly, which reflected the low drought resistance of lettuce at seedling stage. The chlorophyll content, soluble protein content, and soluble sugar content in lettuce seedlings varied greatly under drought stress, which can be used as the main index for further screening of drought-resistant lettuce.

\section{Acknowledgments}

This work was financially supported by the Undergraduate Research Interest Cultivation Project of Sichuan Agricultural University (2020281). 


\section{References}

[1] W. Tian, W. Liu, Y.Z. Song, X. Liu, Effects of Compound Microbial Fertilizer on Growth and Development of Protected Lettuce and Soil Properties, Journal of Qingdao Agricultural University (natural science edition). 36 (2019) 260-266.

[2] Y. Pei, B.C. Zhang, Z.L. Bie, J.H. Zhou, et al., Effect of Trace Irrigation on Growth, Yield and Water Use Efficiency of Lettuce. Northern Horticulture. 13 (2013) 51-53.

[3] J.D. Scharwies, Water transport, perception, and response in plants. J Plant Res. 132 (2019) 311-324.

[4] Y.Y. An, Z.S. Liang, Staged strategy of plants in response to drought stress. Ying Yong Sheng Tai Xue Bao. 23 (2012) 2907-2915.

[5] Q. Li, Y.Z. Qin, W.X. Wang, X.X. Hu, et al., Studies on Physiological Characteristics and Drought Tolerance of Potato (Solanum tuberosum L.) under Drought Stress. Molecular Plant Breeding. 2019 1-14.

[6] J.C. Liu, Z.C. Zhong, Y.J. He, Effects of drought stress and re-watering on the active oxygen scavenging system of Cupressus funebris seedlings in Karst area, Yingyong Shengtai Xuebao (Chinese Journal of Applied Ecology). 22 (2011) 2836-2840.

[7] J.H. Wang, X.M. Zhang, A. Chen, et al., Response of physiological characteristics and anatomical structure of roots in Amorpha fruticosa seedlings exposed to simulated drought with PEG-6000. Acta ecologica sinica. 38 (2018) 511-517.

[8] X.J. Yue, D.P. Quan, T.S. Hong, et al., Non-destructive hyperspectral measurement model of chlorophyll content for citrus leaves. Journal of agricultural engineering, 31 (2015) 294-302.

[9] C.L. Ma, M.Z. Yao, X.C. Wang, et al., Cloning and Expression of Three Genes Involved in Chlorophyll Biosynthesis at Different Albescent Stages of Tea Plant Variety "Baiye 1". Acta Agronomica Sinica. 41 (2015) 240-250.

[10] J. Niu, S.P. Zhang, S.D. Liu, et al., The compensation effects of physiology and yield in cotton after drought stress. Journal of Plant Physiology, 1 (2018) 30-48.

[11] S.K. Dong, K. Zhao, L.J. Liu, Effect of Drought Stress on Chlorophyll Content and Root Activity of Spring Soybean. Soybean science. 30 (2011) 949-953.

[12] A. Arunyanark, S. Jogloy, C. Akkasaeng, et al., Chlorophyll Stability is an Indicator of Drought Tolerance in Peanut. Journal of Agronomy and Crop Science, 194 (2008) 113-125.

[13] W. Xu, K. Cui, A. Xu, et al., Drought stress condition increases root to shoot ratio via alteration of carbohydrate partitioning and enzymatic activity in rice seedlings. Acta Physiologiae Plantarum. 37 (2015) 1-11. 